\title{
Pigeons see the Ponzo illusion
}

\author{
KAZUO FUЛTA \\ Kyoto University, Inuyama, Aichi, Japan \\ and Brown University, Providence, Rhode Island \\ and \\ DONALD S. BLOUGH and PATRICIA M. BLOUGH \\ Brown University, Providence, Rhode Island
}

\begin{abstract}
Three experiments were performed to address factors contributing to the Ponzo illusion. In Experiment 1 , pigeons learned to peck at the longer of two bars in varying line contexts. When these lines converged, the birds had difficulty in learning several patterns in which a Ponzo illusion would reduce the perceived difference. In Experiment 2, the subjects chose one response if a stimu. lus bar was longer than a predetermined length and a second response for shorter bars. The subjects were more likely to choose "long" as the stimulus bar approached the apex of converging lines. These results suggested that pigeons experience the Ponzo illusion. In Experiment 3, the effects of contexts that did and did not form a texture gradient were compared. The magnitude of the illusion did not differ according to context lines. This result failed to support a perspective theory of the Ponzo illusion.
\end{abstract}

Studies of form perception in nonhuman species have demonstrated both similarities and dissimilarities with form perception in humans. For example, judgments about letter similarity are alike in pigeons, nonhuman primates, and humans (Blough, 1982, 1985; Matsuzawa, 1985). On the other hand, recent evidence indicates that pigeons and humans respond differently to certain features of forms in visual search tasks (Allan \& Blough, 1989).

An interesting aspect of pattern perception concerns geometric illusions. Although many researchers have been interested in illusory perception by humans and many different geometric illusions have been discovered, there are few reports of illusory effects in nonhuman animals. Several studies suggest that monkeys are susceptible to geometric illusions: Dominguez (1954) showed that monkeys experience a horizontal-vertical illusion in which vertical lines are judged to be longer than horizontal lines of the same length; Dominguez (1954) and Harris (1968, cited in Davis, 1974) demonstrated that monkeys are likely to see rectangles as being taller than squares (breadth-ofrectangles illusion); Benhar and Samuel (1982) showed that anubis baboons see a Zöllner illusion, in which parallel lines appear to be nonparallel because of the presence of oblique transverse lines; Bayne and Davis (1983) demonstrated that rhesus monkeys are suscepti-

This study was conducted during the first author's stay at Brown University as a visiting scholar, sponsored by the Fellowship Program for Japanese Scholars and Researchers to Study Abroad from the Japan Ministry of Education, Science, and Culture. The study was also supported by NSF Grant BNS 88-19876 to the second and the third authors. Correspondence may be addressed to Kazuo Fujita, Department of Psychology, Primate Research Institute, Kyoto University, Kanrin, Inuyama, Aichi, 484, Japan, or to c42626@kuduts.kudpc.kyoto-u.ac.jp via E-mail. ble to the Ponzo illusion, in which the position of bars within converging lines influences their perceived length.

In the case of pigeons, three studies have addressed the Müller-Lyer illusion. Malott, Malott, and Pokrzywinski (1967) and Malott and Malott (1970) trained birds to respond on a horizontal bar of a particular length with two vertical lines on both ends. They were subsequently tested with bars of various lengths, which had inward or outward arrows on both ends. For the figures with the inward arrows, response rate peaked at a value greater than the training length, as would be expected for the illusion. However, for the figures with the outward arrows, response rate peaked at the same length as that used in the training figure. Thus, the evidence for the illusion was ambiguous. In an unpublished study, Glauber (1986) also obtained some evidence consistent with the illusion. Five pigeons were trained to classify the length of bars into five categories. In subsequent tests with Müller-Lyer figures, 3 of the birds classified figures with inward arrows as longer and figures with outward arrows as shorter than they actually were. However, the other 2 pigeons did not show the effect.

A difficulty in demonstrating the Müller-Lyer illusion in the pigeon may result from the bird's tendency to judge the overall length of bars including arrowheads. In the present study, we addressed another illusion, the Ponzo illusion. In a typical Ponzo illusion figure, two parallel bars are located between two converging lines. The bar near the apex of the context lines looks longer than the bar farther from the apex. The above-mentioned difficulty with the Müller-Lyer figure does not seem to apply to this illusion.

In the present study, we used two different methods to ask whether or not pigeons perceive Ponzo illusions (Ex- 
periments 1 and 2). We further examined the effect of varying contexts on the magnitude of illusion (Experiment 3 ).

\section{EXPERIMENT 1}

In Experiment 1, pigeons learned a simultaneous discrimination of the length of bars located between convergingline contexts. If the pigeons perceive the illusion, their discrimination performance should be affected by the direction of converging contexts, depending upon whether the illusion would increase the perceived difference in bar length or decrease it.

\section{Subjects}

\section{Method}

The subjects were 3 male Carneaux pigeons (Columba livia) housed in separate cages. Their body weights were kept at approximately $80 \%$ of their free-feeding weights throughout the experiment. The birds had participated in several visual discrimination experiments unrelated to the present study.

\section{Apparatus}

Three experimental boxes $(31.5 \mathrm{~cm}$ wide, $36 \mathrm{~cm}$ high, and $30.5 \mathrm{~cm}$ deep) were located in a dark room. The front wall of each box contained a rectangular opening (11 cm wide and $8.5 \mathrm{~cm}$ high). Its lower edge was $18.5 \mathrm{~cm}$ above the floor. A 5 -in. color video monitor (Rhapsody TV-670) was mounted $2.5 \mathrm{~cm}$ behind the opening; the screen was surrounded by a frame that held infrared-beam response detectors (Carol Touch). A second opening, $13.5 \mathrm{~cm}$ below the opening of the screen, provided access to a food tray and could be illuminated by a 3-W tungsten bulb. Personal computers (Atari 800 ) presented graphic patterns on the TV as stimuli and controlled the equipment. White noise masked external noise.

\section{Stimuli and Procedure}

The stimuli were white graphic patterns displayed on the TV. In the subsequent part of this paper, we will give their sizes in terms of the number of graphic dots, or pixels, where appropriate, as well as the actual lengths on the screen in millimeters. On the TV screen, a line composed of 100 pixels measured $32 \mathrm{~mm}$ horizontally and $37 \mathrm{~mm}$ vertically.

In the following four phases, the pigeons' task was to peck at the longer of two bars. After intertrial intervals of $5 \mathrm{sec}$, two vertical bars of different lengths appeared at the center of the TV screen. The location of the positive bar was quasirandomly assigned to the right or the left, trial by trial. Completion of a specified peck sequence defined a response. When directed to the longer bar, a response produced reinforcement, which could be either the flash of the magazine light alone or the flash accompanied by the raising of the food magazine for predetermined durations, adjusted so that individuals maintained their body weights during sessions. The probability of food reinforcement varied from $100 \%$ to $20 \%$, depending on the phases and individuals. A response to the shorter bar was followed by a 5-sec darkening of the screen.

The experimental conditions in the following four phases are summarized in Table 1.

Phase 1: Discrimination of isolated bars. In the first stage of this phase, the stimuli were pairs of white vertical bars, $7.4 \mathrm{~mm}$ (20 pixels) and $11.1 \mathrm{~mm}$ (30 pixels) long; the bars in each pair were separated by $12.8 \mathrm{~mm}$ (40 pixels). Both bars were $1.6 \mathrm{~mm}$ ( 5 pixels) wide (Figure 1, panel a, left). The required peck sequence, the probability of food reinforcement, and the number of trials per session varied, depending on the phases and the subjects. These are listed in Table 1. Training was continued until the subjects reached above $85 \%$ correct for two consecutive sessions.

After the subjects reached this criterion, they were trained to discriminate between new bars with lengths of $3.7 \mathrm{~mm}$ (10 pixels) and $7.4 \mathrm{~mm}$ (20 pixels) (Figure 1, panel a, right). Sessions included trials with this and the 7.4/11.1 pair. The 3.7/7.4 and 7.4/11.1 pairs appeared randomly, but equally often. Training was continued until the subjects reached the training criterion.

Phase 2: Discrimination of bars between parallel context lines. Two horizontal parallel lines $29.4 \mathrm{~mm}$ (92 pixels) long were added to the patterns of Phase 1 (Figure 1, panel b, upper two patterns). These lines were $0.7 \mathrm{~mm}$ ( 2 pixels) thick and were separated vertically by $16.3 \mathrm{~mm}$ (44 pixels). As in Phase 1, the $3.7 / 7.4$ and

Table 1

Experimental Conditions in Each Phase of Experiment 1

\begin{tabular}{|c|c|c|c|c|c|c|}
\hline Subject & Bar Length & Context & $\begin{array}{c}\text { Peck } \\
\text { Sequence }\end{array}$ & $\%$ Food & $\begin{array}{c}\text { Trials per } \\
\text { Session } \\
\end{array}$ & $\begin{array}{c}\text { No. } \\
\text { Sessions }\end{array}$ \\
\hline \multicolumn{7}{|c|}{ Phase 1} \\
\hline $\begin{array}{l}544 \\
942 \\
936\end{array}$ & $\begin{array}{l}7.4,11.1 \rightarrow 3.7,7.4,11.1 \\
7.4,11.1 \rightarrow 3.7,7.4,11.1 \\
7.4,11.1 \rightarrow 3.7,7.4,11.1\end{array}$ & $\begin{array}{l}\text { None } \\
\text { None } \\
\text { None }\end{array}$ & $\begin{array}{l}1 \rightarrow 3 \\
1 \rightarrow 3 \\
1 \rightarrow 3\end{array}$ & $\begin{array}{l}100 \\
100 \\
100\end{array}$ & $\begin{array}{l}96 \\
96 \\
96\end{array}$ & $\begin{array}{l}14 \\
26 \\
13\end{array}$ \\
\hline \multicolumn{7}{|c|}{ Phase 2} \\
\hline $\begin{array}{l}544 \\
942 \\
936\end{array}$ & $\begin{array}{l}3.7,7.4,11.1 \\
3.7,7.4,11.1 \\
3.7,7.4,11.1\end{array}$ & $\begin{array}{l}\text { Par } \\
\text { Par } \\
\text { Par }\end{array}$ & $\begin{array}{l}3 \\
3 \\
4\end{array}$ & $\begin{array}{l}100 \rightarrow 20 \\
100 \rightarrow 50 \\
100 \rightarrow 50\end{array}$ & $\begin{array}{l}96 \rightarrow 480 \\
96 \rightarrow 192 \\
96 \rightarrow 192\end{array}$ & $\begin{array}{l}28 \\
17 \\
25\end{array}$ \\
\hline \multicolumn{7}{|c|}{ Phase 3} \\
\hline $\begin{array}{l}544 \\
942 \\
936\end{array}$ & $\begin{array}{l}3.7,7.4,11.1 \\
3.7,7.4,11.1 \\
3.7,7.4,11.1\end{array}$ & $\begin{array}{l}\text { Par }+ \text { Cnv } \\
\text { Par }+ \text { Cnv } \\
\text { Par }+ \text { Cnv }\end{array}$ & $\begin{array}{l}3 \\
3 \\
4\end{array}$ & $\begin{array}{l}20 \\
50 \\
50\end{array}$ & $\begin{array}{l}480 \\
192 \\
192\end{array}$ & $\begin{array}{l}2 \\
2 \\
2\end{array}$ \\
\hline \multicolumn{7}{|c|}{ Phase 4} \\
\hline $\begin{array}{l}544 \\
942 \\
936\end{array}$ & $\begin{array}{l}5.6,7.4,9.3 \\
5.6,7.4,9.3 \\
5.6,7.4,9.3\end{array}$ & $\begin{array}{l}\text { Par }+ \text { Cnv } \\
\text { Par+Cnv } \\
\text { Par+Cnv }\end{array}$ & $\begin{array}{l}3 \\
3 \\
4\end{array}$ & $\begin{array}{c}20 \\
50 \rightarrow 33 \\
50 \rightarrow 33\end{array}$ & $\begin{array}{l}480 \\
288 \\
288\end{array}$ & $\begin{array}{l}6 \\
6 \\
6\end{array}$ \\
\hline
\end{tabular}

Note-Bar lengths are in millimeters. Context refers to the type of context lines-parallel (Par) or converging (Cnv). Peck Sequence refers to required peck sequence on the stimuli. \%Food is the probability of food reinforcement. 
a

b
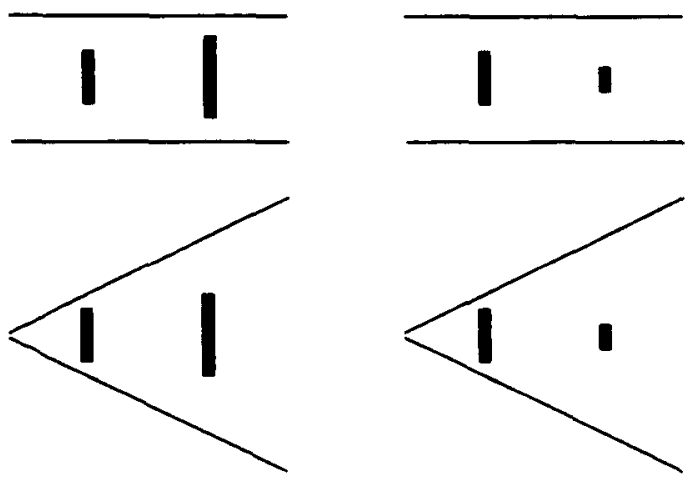

C
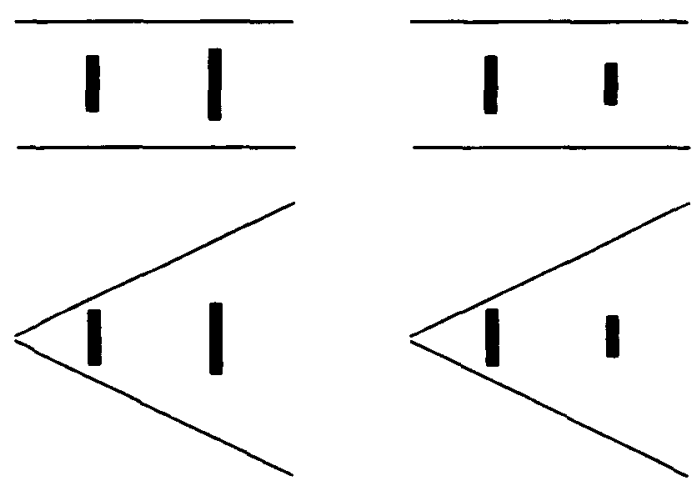

Figure 1. Examples of stimuli used in Experiment 1. (a) Stimuli used in Phase 1. (b) Stimuli used in Phases 2 and 3. (c) Stimuli used in Phase 4. The actual stimuli were white on a black background. (See text for details.)

7.4/11.1 pairs appeared equally often. Training was continued until the subjects reached the training criterion.

Phase 3: Easy discrimination of bars between parallel and converging context lines. Patterns with two lines converging either rightward or leftward were added (Figure 1, panel b, lower two patterns). The lines were $0.7 \mathrm{~mm}$ ( 2 pixels) thick, and their horizontal length was $29.4 \mathrm{~mm}$ (92 pixels). The midpoints of these lines were separated by $16.3 \mathrm{~mm}$ ( 44 pixels). When the converging lines appeared, the stimulus bar near the apex was always $7.4 \mathrm{~mm} \mathrm{(20}$ pixels) long. Patterns with parallel lines and those with converging lines were presented equally often, as were the 3.7/7.4 and 7.4/11.1 bar pairs. Training was continued until the subjects reached the training criterion.

Phase 4: Difficult discrimination of bars between parallel and converging context lines. The lengths of the stimulus bars were changed to $5.6,7.4$, and $9.3 \mathrm{~mm}(15,20$, and 25 pixels, respectively) (Figure 1, panel c). As in the previous phases, one of the bars was always $7.4 \mathrm{~mm}$ (20 pixels) long, and it occupied the location near the apex of the converging lines. Training terminated after six sessions.

\section{Results and Discussion}

The number of sessions required to reach the training criterion is summarized in the last column of Table 1. All pigeons acquired the discrimination as far as Phase 3. In Phase 4, however, in which the difference in bar length decreased, accuracy dropped for all subjects. The learning functions for this phase are shown in Figure 2.

In the parallel-line context, accuracy was in the $60 \%-80 \%$ range, and it did not appear to depend on the bar pair (top panel). However, in the converging-line context (bottom panel), accuracy increased for the 7.4/5.6 bar pair and diminished for the 7.4/9.3 bar pair. Two separate one-factor randomized-block analyses of variance, one for parallel context and the other for converging context, addressed this dissociation. Sessions were taken as randomized blocks. For patterns with parallel context, no bird showed a significant difference in the effect of the combination of bars $[F(1,5)=1.55$ for Pigeon 544; $F(1,5)=1.16$ for Pigeon 942; $F(1,5)=0.76$ for Pigeon 936]. However, for patterns of converging context, all 3 subjects showed significantly higher accuracy in $7.4 / 5.6$ trials than in $7.4 / 9.3$ trials $[F(1,5)=$ 7.46, $p<.05$, for Pigeon 544; $F(1,5)=26.10, p<$ .01 , for Pigeon $942 ; F(1,5)=17.06, p<.01$, for Pigeon 936]. It should be recalled that the location near the apex of the converging lines was always occupied by the 7.4-mm bar. That is, pigeons had difficulty in choosing the physically longer bar when it was located farther from the apex (see Figure 1, panel c, bottom left). The pigeons seem to have perceived bars near the apex of the converging lines as being longer than those farther from the apex. This outcome showed preliminary evidence for the Ponzo illusion.

\section{EXPERIMENT 2}

In Experiment 2, a successive discrimination method was used to obtain psychophysical functions for bar length. Since the Ponzo illusion changes perceived length, the converging-line context should modify the position of this function.

\section{Method}

Subjects and Apparatus

The subjects and the apparatus were the same as in Experiment 1.

\section{Stimuli and Procedure}

The stimuli were changed to black graphic patterns on a white background, because these pictures were sharper than white on black.

After an intertrial interval of $5 \mathrm{sec}$, a horizontal bar appeared at the center of the screen with or without a line context, depending on the phase. After five pecks at the bar, two choice response locations appeared at the bottom corners of the screen with the sample bar still present. The right choice was marked by a filled rectangle of $5.9 \times 6.4 \mathrm{~mm}(16 \times 20$ pixels $)$ and the left choice by an open rectangle of the same size. The choice locations were assigned to "long" and "short" bars, with different assignments for different subjects. A single response to the assigned location was a correct choice and was reinforced. Errors produced a 5-sec darkening of 

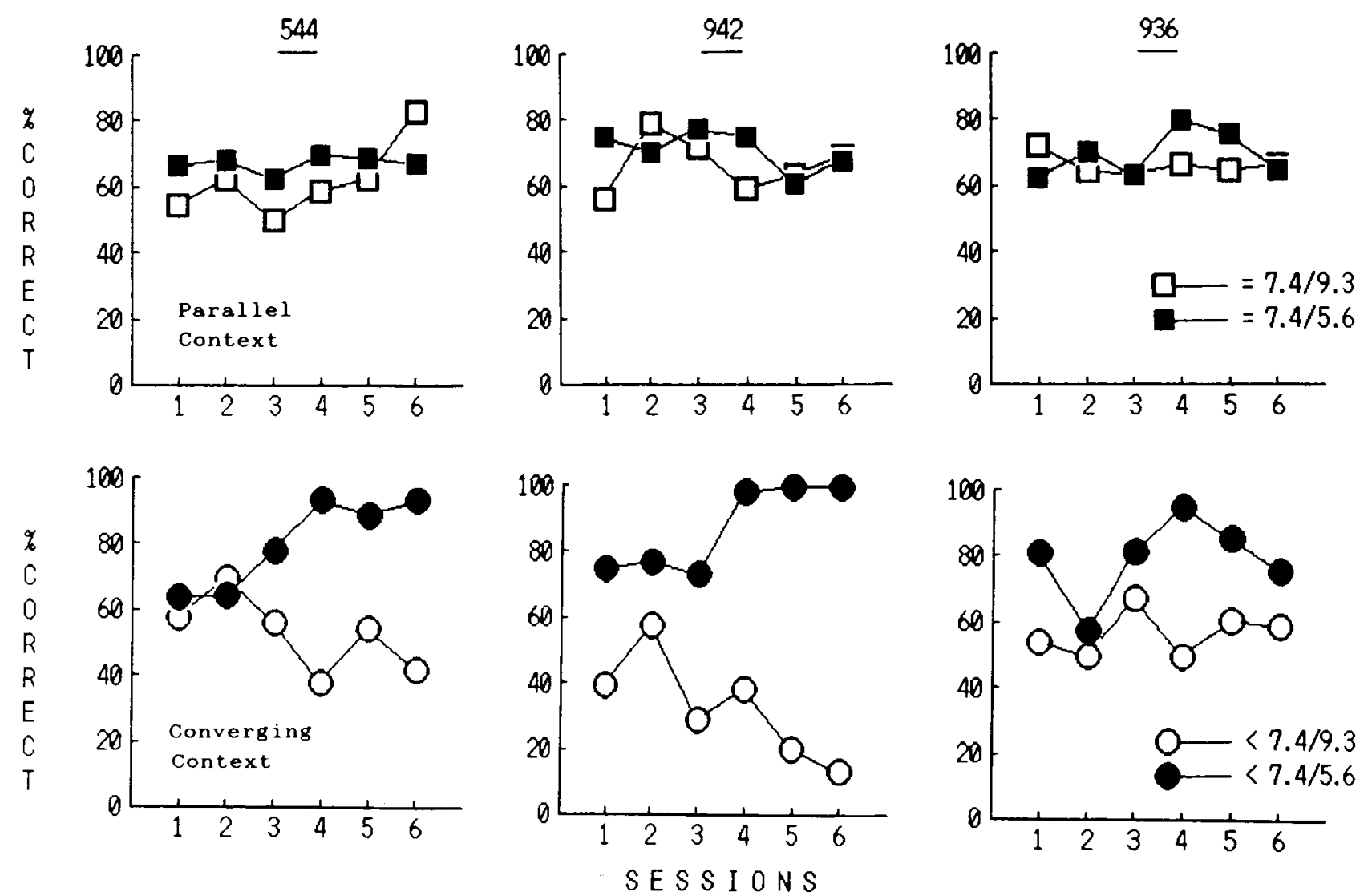

Figure 2. The learning curve of each individual bird in Phase 4 of Experiment 1. The horizontal axis is the number of sessions and the vertical axis is the percent correct responses. Top panel: patterns containing parallel context lines (see the upper two patterns of panel $\mathrm{c}$ in Figure 1). Bottom panel: patterns with converging context lines (see the lower two patterns of panel $c$ in Figure 1). Unfilled symbols designate discrimination between bars of 7.4 and $9.3 \mathrm{~mm}$; filled symbols designate discrimination between bars of 7.4 and $5.6 \mathrm{~mm}$.

the screen. The probability of food reinforcement was varied from $100 \%$ to $16 \%$ in the training sessions.

The experimental conditions of the following four phases are summarized in Table 2.

Phase 1: Preliminary training. The stimuli were the two horizontal bars $2.6 \mathrm{~mm}$ (8 pixels) and $6.4 \mathrm{~mm}$ (20 pixels) long and $1.1 \mathrm{~mm}$ ( 3 pixels) thick. The probability of food reinforcement and the number of trials per session are listed in Table 2 . Training was continued until the subjects had reached $80 \%$ correct in two successive sessions. A correction method, in which the same trial was repeated until the subjects made a correct response, was employed after two sessions for Pigeon 544 and after three sessions for the other 2. Data from correction trials were not used in accuracy computations.

Phase 2: Baseline training. In this phase, the stimuli were bars of length $3.2,3.8,4.5,5.1,5.8$, or $6.4 \mathrm{~mm}(10,12,14,16,18$, or 20 pixels, respectively), in contexts made up of vertical parallel lines. The stimulus arrangement is illustrated in Figure 3 (left). There were four lines on each side of the stimulus bar. The lines were $30.3 \mathrm{~mm}$ ( 82 pixels) long and $0.6 \mathrm{~mm}$ (2 pixels) thick. The two inner lines were separated from each other by $7.7 \mathrm{~mm}(24$ pixels), and the remaining lines were separated by $3.8 \mathrm{~mm}(12$ pixels). The position of the context lines varied; their upper ends could be $24.4,17.0$, or $9.6 \mathrm{~mm}(66,46$, or 26 pixels) above the top of the horizontal bar, to produce high-, middle-, or low-context patterns, respectively.
Reinforcement was contingent on choices associated with "long" for the 5.1-, 5.8-, and 6.4-mm bars and "short" for the 3.2-, 3.8-, and $4.5-\mathrm{mm}$ bars. The training was continued until the subjects had reached $80 \%$ correct for two consecutive sessions.

Phase 3: One-point test. Three new test stimulus patterns were prepared. They are illustrated in Figure 3 (right). The innermost lines were separated by $1.9 \mathrm{~mm}$ (6 pixels) at the top and $19.2 \mathrm{~mm}$ (60 pixels) at the bottom ends. The remaining lines were separated by $1.0 \mathrm{~mm}$ ( 3 pixels) from each other at the top and $9.6 \mathrm{~mm}$ (30 pixels) at the bottom. The $4.8-\mathrm{mm}$ (15-pixel) bar appeared in the parallel-line context, as in Phase 2, or the converging-line context, each context having the three locations specified above (low, middle, and high). When the converging context was presented in the middle location, the distance between the two innermost bars at the height of the horizontal bar was $7.7 \mathrm{~mm}$ (24 pixels), and the remaining lines were separated from each other by $3.8 \mathrm{~mm} \mathrm{(12}$ pixels), which was the same separation as that for the parallel lines. Note that the distance between the stimulus bars and context lines varied according to bar length and context position. However, the stimulus bar never touched the context lines. The separation was the smallest, $0.3 \mathrm{~mm}$ ( 1 pixel), when the $6.4-\mathrm{mm}$ bar was presented in low-context lines.

Sessions consisted of 576 trials, in which all Phase 2 patterns as well as the additional three test patterns described above appeared in a quasirandom order. The probability of food reinforcement was $80 \%$ of the value used in the previous training phase. For the test 
Table 2

Experimental Conditions in Each Phase of Experiment 2

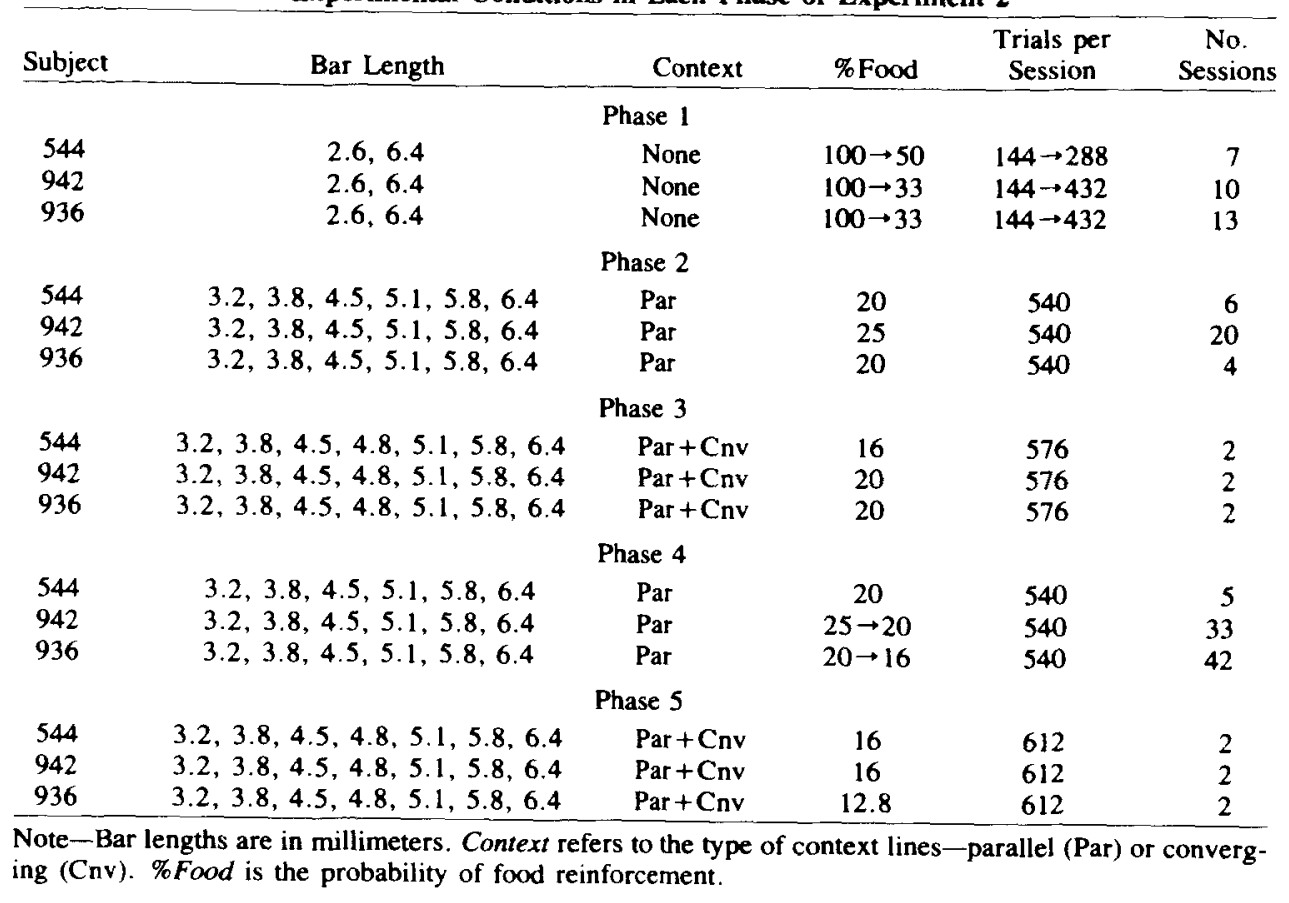

Training Patterns

Low Context
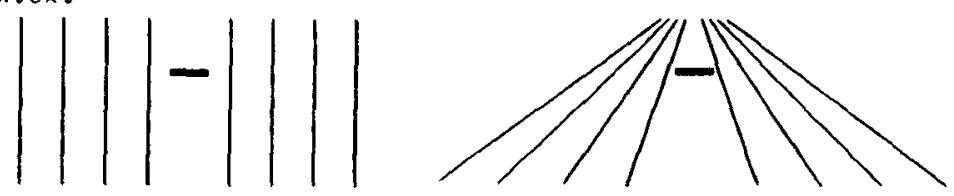

niddle context
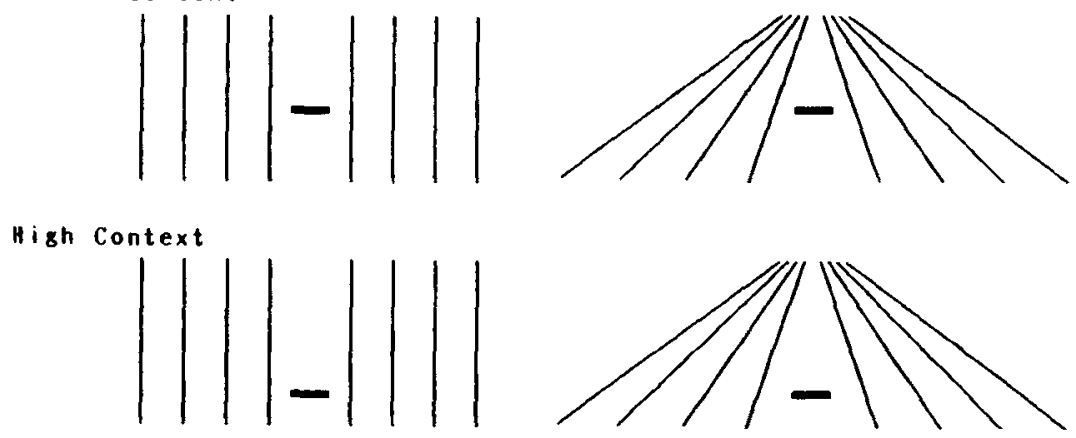

Figure 3. Examples of stimuli used in Experiment 2. The left three patterns are the training stimuli used in Phases 2-5, and the right three patterns are the test stimuli used in Phases 3 and 5. (See text for details.) 
patterns, responses were reinforced without regard to the choice. The differential reinforcement contingency was in effect for the other baseline trials.

Phase 3 occurred in two sessions, separated by one session of Phase 2 baseline training. Overall, there were 48 trials at each test pattern.

Phase 4: Baseline Training 2. Immediately after Phase 3, baseline training resumed as in Phase 2 . Training continued until the subjects had reached $80 \%$ correct in two consecutive sessions.

Phase 5: Five-point test. Fifteen test stimuli with convergingline contexts were prepared. The characteristics of the converging lines were the same as in Phase 3 . The bars of these test stimuli were $3.8,4.5,4.8,5.1$, or $5.8 \mathrm{~mm}(12,14,15,16$, or 18 pixels) long. As in Phase 3, the context lines were presented in the three locations: low, middle, and high.

The test sessions consisted of 612 trials, including 12 trials per test pattern. As in Phase 3, all the choice responses were nonselectively reinforced in the test trials. The contingencies in the baseline trials were the same as in training. The probability of primary reinforcement was $80 \%$ of that in Phase 4 .

The test was conducted for two sessions, separated by three baseline sessions. Altogether, 24 trials per test pattern were run.

\section{Results and Discussion}

The number of training sessions is listed in Table 2. The pigeons mastered the baseline training of Phase 2 quickly, except that Pigeon 942 did not reach the training criterion in 20 sessions. His final accuracy was about $70 \%$ correct. This pigeon also concluded Phase 4 after 33 sessions. His final accuracy was about $75 \%$.

In Figure 4, the results of the one-point test in Phase 3 are shown for each pigeon. In the parallel-line contexts, all pigeons showed an increase of the proportion of "long" choices with increasing bar length for all three context positions, although Pigeon 942 performed somewhat inconsistently.

For the converging-line contexts, the proportion of "long"' choices was the highest in the low-context condition (circles) and the lowest in the high-context condition (squares) for all 3 subjects. These proportions differed significantly from those expected by chance for all the pigeons $\left[\chi^{2}(2, N=144)=26.89, p<.01\right.$, for Pigeon 544; $\chi^{2}(2$, $N=144)=9.65, p<.01$, for Pigeon 942; $\chi^{2}(2, N=144)$ $=12.54, p<.01$, for Pigeon 936]. With the low-context positions, the stimulus bar was located nearer the apex of the converging lines. Thus this outcome is consistent with the Ponzo illusion, whereby bars are perceived to be longer as they approach an apex.

In Figure 5, the results of the five-point test in Phase 5 are shown for each pigeon. The proportion of "long" choices was an increasing function of bar length for both parallel-line contexts and converging-line contexts, though data for the latter were more irregular. For the convergingline contexts, Pigeon 544 showed the highest choice proportion of "long"' in the low-context condition (circles) and the lowest in the high-context condition (squares). The other 2 pigeons showed similar trends. A two-factor analysis of variance was used to evaluate bar length and context location as fixed factors. Scores from the two test sessions were entered as the error term. The main effect of context location was significant for all birds $[F(2,15)=$ $84.15, p<.01$, for Pigeon 544; $F(2,15)=23.20, p<$ .01 , for Pigeon $942 ; F(2,15)=4.27, p<.05$, for Pigeon 936]. The main effect of bar length was also significant for Pigeons 544 and 942 and was almost significant for Pigeon $936[F(4,15)=21.70, p<.01, F(4,15)$
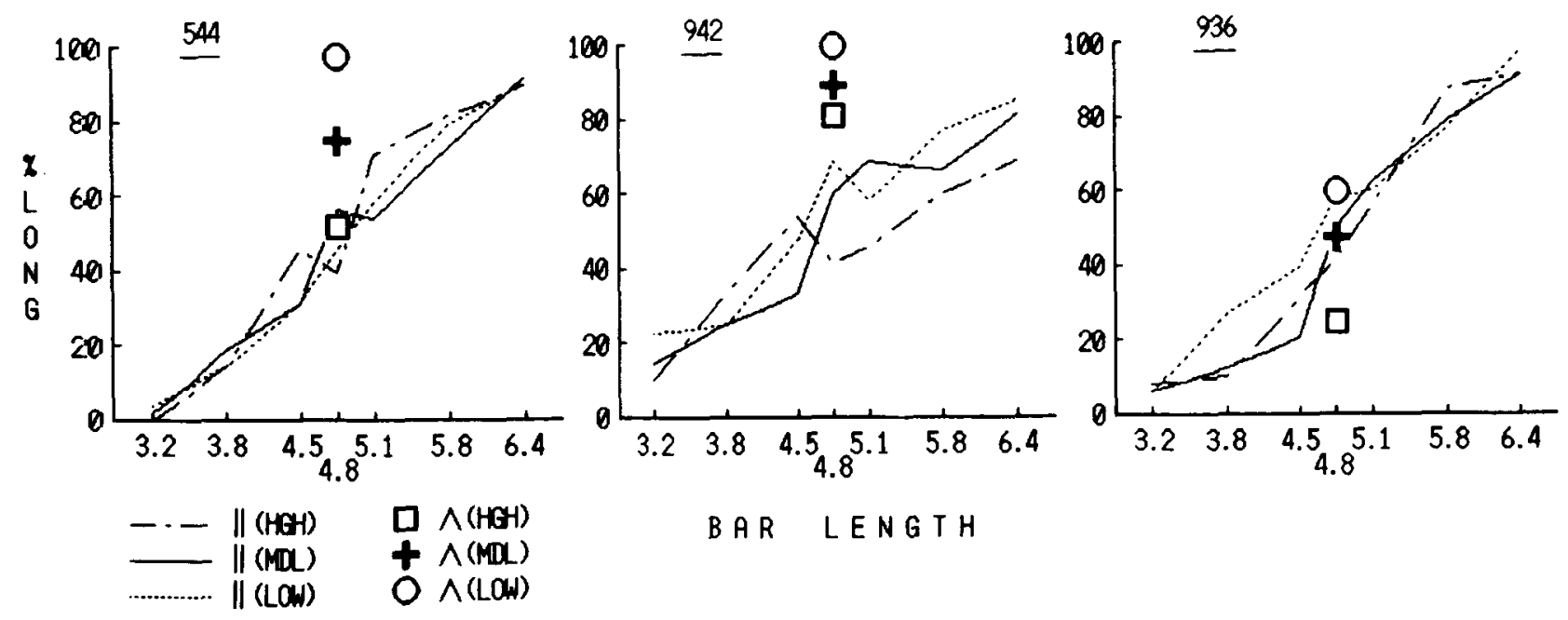

BAR LENGTH

Figure 4. The results of the one-point test for each bird in Phase 3 of Experiment 2. The vertical axis is the percent of "long" key choices and the horizontal axis is the length of the stimulus bars. The lines without symbols are from the training stimuli (see the left patterns in Figure 3) and the isolated symbols are from the test stimuli (see the right patterns in Figure 3). The squares represent high-context patterns, the pluses represent middle-context patterns, and the circles represent low-context patterns. 

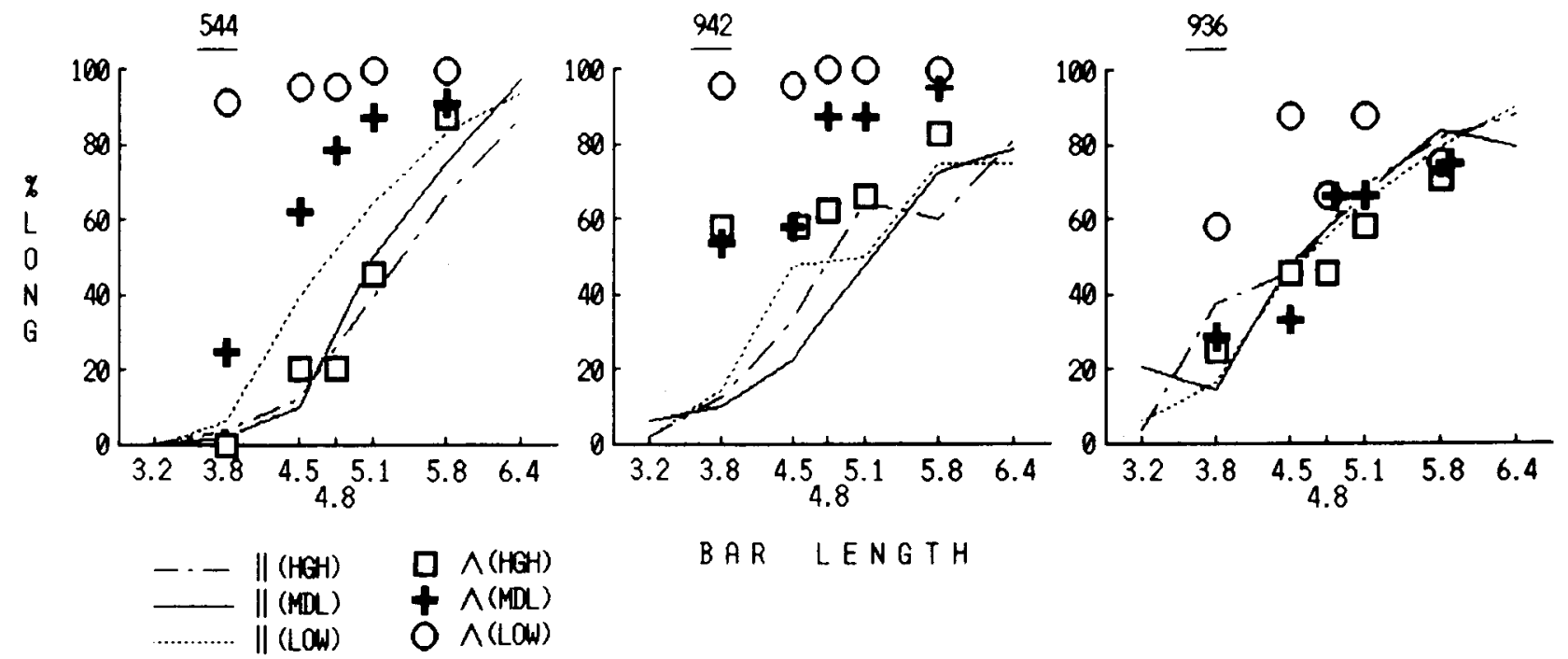

Figure 5. The results of the five-point test for each bird in Phase 5 of Experiment 2. Other details are as in Figure 4.

$=5.07, p<.01$, and $F(4,15)=2.82, p<.10$, respectively]. The results clearly demonstrated that the pigeons perceived the bars near the apex of the convergingline contexts as being longer than those that were far from it. Thus the results are consistent with the Ponzo illusion.

\section{EXPERIMENT 3}

Experiments 1 and 2 suggested that pigeons and humans perceive the Ponzo illusion in similar ways, although this similarity does not demonstrate that the same mechanisms are responsible. The literature on humans offers at least two theories that may account for the illusion: perspective theory (Gregory, 1963) and assimilation theory (Pressey, 1967, 1971).

In perspective theory, it is assumed that when subjects see the two-dimensional illusory figures, they unconsciously perceive depth in the figure. In the Ponzo figures, the bars near the apex of the converging lines are perceived as being more distant. As a result, the subjects adjust the lengths of the bars to maintain size constancy, and hence the bars near the apex are judged to be longer.

In assimilation theory, there are two major postulates: "(1) Whenever judgments are made of a series of magnitudes, the smaller magnitudes in that series will be overestimated and the larger magnitudes will be underestimated. (2) Other things being equal, a context which falls within the attentive field will be more effective than a context outside that field" (Pressey, 1971, p. 172). The application of this theory to the Ponzo illusion is illustrated in Figure 6. When subjects see the Ponzo figure in the left panel, the parts of the figure within the round attentive field (right panel) are particularly influential. The upper bar is assimilated to the distances between context lines below it, and hence it is perceived to be longer than the lower bar.
Though perspective theory appears to explain many illusory figures, several studies on human perception have failed to support it. For instance, when the bars lie in the same direction as the converging lines, very little illusion is seen (Humphrey \& Morgan, 1965). Stereoscopic presentation of Müller-Lyer figures does not enhance the illusion as perspective theory predicts it should (Georgeson \& Blakemore, 1973). A texture analogue of converging lines did not produce as good illusions as did actual lines (Fineman \& Carlson, 1973). A Ponzo figure that was put in a background drawing in a way that did not suggest depth produced the same amount of illusion as did the isolated Ponzo figure (Newman \& Newman, 1974).

In Experiment 3, we compared the amounts of illusion perceived by pigeons for two types of context: In one, the converging lines formed a texture gradient, and in the other, they did not. To human eyes, the former gave a stronger perception of depth than the latter did. The innermost lines were identical in the two conditions. Perspective theory predicts that the gradient-forming lines will

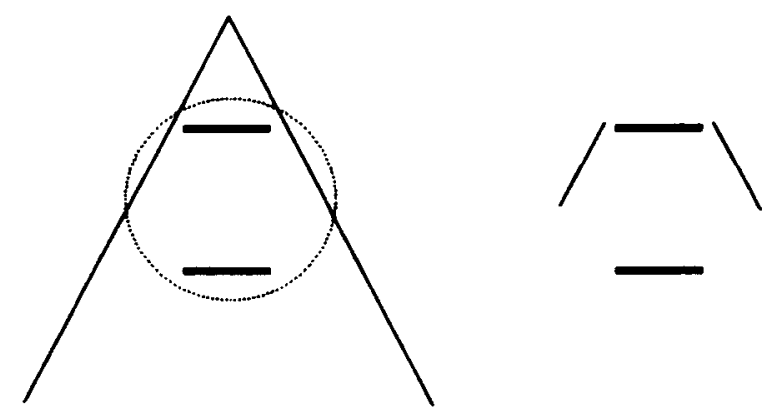

Figure 6. Explanation of the Ponzo illusion according to the assimilation theory presented by Pressey (1971). (See text for details.) 
yield a greater illusion because of the stronger depth perception, whereas assimilation theory predicts little difference as long as the innermost lines are the same.

\section{Method}

\section{Subjects and Apparatus}

The same 3 subjects served immediately after Experiment 2 in the same apparatus.

\section{Stimuli}

The stimuli are illustrated in Figure 7. Half of the patterns included the same converging lines as those in Experiment 2 (left column). We will refer to this type of context as the perspective context. The other half included four parallel slanted lines, separated by $3.8 \mathrm{~mm}$ ( 12 pixels), on each side of the bar (right column). The innermost lines were exactly the same as the perspective context. We will refer to this type of context as the nonperspective context.

\section{Procedure}

Phase 1: Baseline training. The method from Experiment 2 was used to train all 3 pigeons on successive discriminations of bar length with perspective context and nonperspective context. One choice ("short') was reinforced when the bar was $3.2,3.8$, or $4.5 \mathrm{~mm}$ $(10,12$, or 14 pixels, respectively) long, and the alternative choice ("long") was reinforced when the bar was $5.1,5.8$, or $6.4 \mathrm{~mm}$ $(16,18$, or 20 pixels) long. All the line contexts were presented in the middle location (see Experiment 2). The perspective lines and nonperspective lines were presented quasirandomly with the same frequency.

The probability of primary reinforcement was $20 \%$ for Pigeons 544 and 942 and $16 \%$ for Pigeon 936. Training continued until the subjects had reached more than $70 \%$ correct for both contexts for two consecutive sessions.
Phase 2: Test. This phase included tests with high- and lowcontext locations. The bar lengths were the same as those in Phase 1. Half of the 576 trials per session used the middle-context condition trained in Phase 1. The other half were test trials in which all the choice responses were reinforced without regard to the choice. Each combination of bar length and the context location was tested on 12 trials.

The probability of food reinforcement was set at $80 \%$ of that of the previous phase. The test was conducted for two sessions separated by at least three baseline sessions.

\section{Results and Discussion}

Phase 1 was mastered in 5 sessions by Pigeon 544 and in 8 sessions by Pigeon 936. Pigeon 942 concluded training after 20 sessions. Although he did not satisfy the criterion, his accuracy averaged more than $70 \%$ for each type of context in the last 3 sessions.

In Figure 8, the percent of "long" choices is shown as a function of length of the stimulus bars for each pigeon. For all stimulus conditions, the proportion of "long" responses increased with length of the stimulus bar. As in Experiment 2, all pigeons chose "long" most frequently in the low-context condition (circles) and least frequently in the high-context condition (squares). A three-factor analysis of variance was used to evaluate bar length, context location, and context type as fixed factors. Scores from the two test sessions were entered as the error term. The main effect of context location was significant for all subjects $[F(2,36)=82.12, p<.01, F(2,36)=67.57$, $p<.01$, and $F(2,36)=15.00, p<.01$, for Pigeons 544, 942, and 936, respectively]. This result again confirmed that the pigeons perceived the Ponzo illusion for
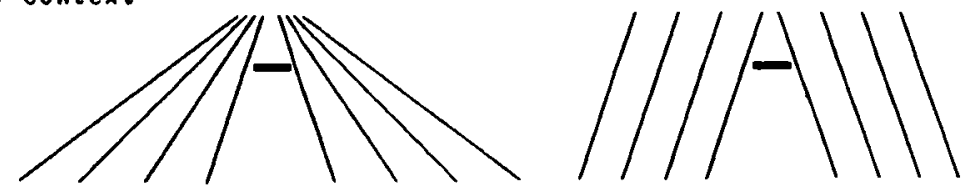

Middle Context
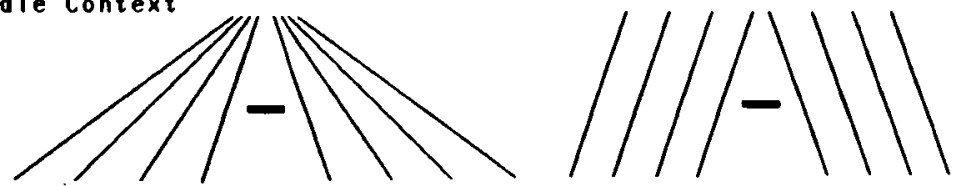

High Context
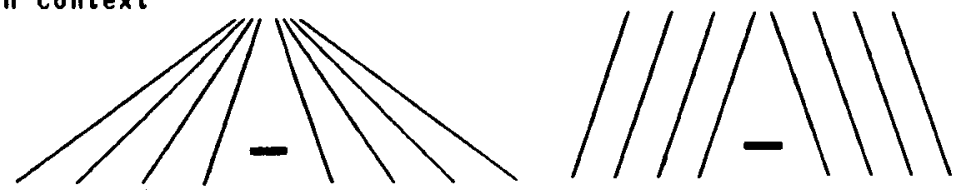

Figure 7. Examples of stimuli used in Experiment 3. The left three patterns are the stimuli with perspective lines; the right patterns are those with nonperspective lines. The middle two patterns (middle context) were used in the training. The other four patterns were the test stimuli. (See text for details.) 

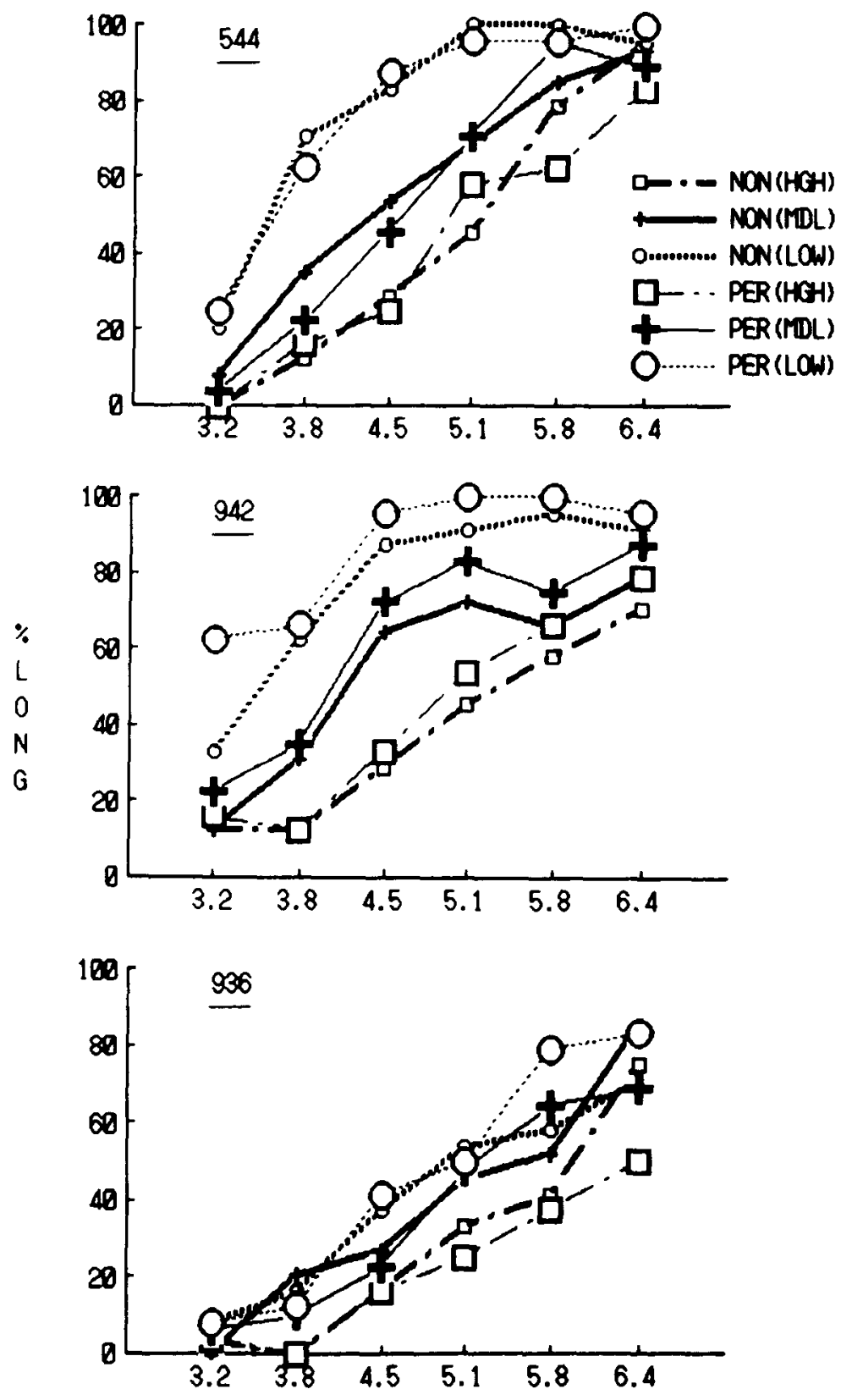

BAR LENGTH

Figure 8. The results of Experiment 3 for each bird. The vertical axis is the percent of "long" key choices; the horizontal axis is the length of the stimulus bars. The thick lines with small symbols are from patterns with nonperspective contexts (left patterns in Figure 7), and the thin lines with large symbols are from those with perspective contexts (right patterns in Figure 7). 
both types of context lines. The main effect of the bar length was also significant $[F(5,36)=127.86, p<.01$, $F(5,36)=43.88, p<.01$, and $F(5,36)=60.57, p<$ .01 , respectively]. The main effect of context type was significant for Pigeon $942[F(1,36)=7.43, p<.01]$, but not significant for Pigeons 544 and $936[F(1,36)=$ 0.82 and $F(1,36)=0.14$, respectively]. However, the interaction of context location and context type was not significant for any of the birds $[F(2,36)=0.09, F(2,36)=$ 0.18 , and $F(2,36)=1.38$, for Pigeons 544, 942, and 936, respectively]. This result did not indicate a difference between perspective and nonperspective contexts in their tendency to produce the Ponzo illusion.

The magnitude of the illusion was calculated in terms of the shift of the subjective neutral point: Percent scores shown in Figure 8 were converted to $Z$ scores. Linear regressions were then calculated for each of the six stimulus conditions and the subjective neutral point interpolated at $Z=0$ (50\% "long" choice). The magnitude of the illusion was the deviation of these neutral points for the high- or low-context condition from that for the middlecontext condition.

In the low-context condition, Pigeons 544, 942, and 936 overestimated the bar length by $34.3 \%, 41.3 \%$, and $1.0 \%$, respectively, for nonperspective contexts. For perspective contexts, the overestimations were $27.6 \%, 70.2 \%$, and $8.9 \%$, respectively. In the high-context condition, the birds underestimated the bar length by $11.1 \%, 14.8 \%$, and $8.0 \%$ for nonperspective contexts, and by $12.1 \%$, $19.0 \%$, and $13.1 \%$ for perspective contexts. A threefactor analysis of variance of context location (high or low) $\times$ context type $\times$ subject was conducted with the three-way interaction as the error term. The main effect of context location was again significant $[F(1,2)=175.0$, $p<.01]$. However, there was neither a main effect of the type of context on the magnitude of the illusion $[F(1,2)=2.91$, n.s. $]$, nor an interaction of the effects of context location and type of context $[F(1,2)=8.95$, n.s.]. The main effect of subject was not significant $[F(2,2)=14.94$, n.s. $]$.

\section{GENERAL DISCUSSION}

In Experiment 1, pigeons had difficulty learning a bar-length discrimination when, by human standards, a converging-line context reduced the perceived difference between the stimuli. In Experiment 2, pigeons showed a systematic distortion in bar-length perception according to the distance between the bar and the apex of this context. This finding was replicated in Experiment 3. These results demonstrate that pigeons perceive the Ponzo illusion.

In Experiment 3, the magnitude of the illusion was compared between contexts that did and did not form texture gradient. The perspective theory predicts that contexts that help perception of subjective depth ought to enhance the illusion. On the other hand, the assimilation theory does not predict an effect of perspective as long as the stimuli that fall in the attentive field remain constant. We did not find a significant effect of perspective. Though 2 of the birds showed somewhat larger illusions for perspective lines, the 3rd bird (544) showed the opposite tendency in low-context conditions. The present results seem to be more favorable to the assimilation account of the pigeon's perception of the Ponzo illusion, if one assumes that the pigeon's attentive field is limited to the innermost two line contexts.

It is difficult, however, to assess the size of the pigeon's attentive field and the extent to which the pigeon uses perspective cues. In keypecking tasks, pigeons maintain a viewing distance of about $5-10 \mathrm{~cm}$. The pattern used in Experiment 3 would thus have subtended $42^{\circ}-75^{\circ}$ of visual angle, and it is not clear that the birds used all of the information from this sizable field. Also, pigeons may not use perspective cues even when they attend to those cues. Cerella (1977) showed that pigeons failed to learn to discriminate perspective line drawings of a cube from nonperspective drawings of the same size. The present results thus do not reject the perspective theory completely.

The fact that pigeons perceive the Ponzo illusion suggests common physiological mechanisms of perception. There are many differences between human and pigeon visual systems; for example, pigeons have a panoramic visual field with a $25^{\circ}$ wide binocular field, an extra area that has many photoreceptors in addition to the fovea, several different types of oil droplets in the retina, complete decussation at the optic chiasm, tectum- rather than cortex-based vision, and so forth (Donovan, 1978). As Blough (1984) pointed out, similarities between pigeons and humans suggest important constraints on neural hypotheses. If pigeons see the Ponzo illusion as humans do, perception of the illusion is unlikely to be attributable to species-unique characteristics of neural organization.

\section{REFERENCES}

Allan, S. E., Blough, D. S. (1989). Feature-based search asymmetries in pigeons and humans. Perception \& Psychophysics, 46, 456-464.

Bayne, K. A. L., \& DAvis, R. T. (1983). Susceptibility of thesus monkeys (Macaca mulatta) to the Ponzo illusion. Bulletin of the Psychonomic Society, 21, 476-478.

Benhar, E., Samuel, D. (1982). Visual illusions in the baboon (Papio anubis). Animal Learning \& Behavior, 10, 115-118.

Blough, D. S. (1982). Pigeon recognition of letters of the alphabet. Science, 218, 397-398.

BLough, D. S. (1984). Form recognition in pigeons. In H. L. Roitblat, T. G. Bever, \& H. S. Terrace (Eds.), Animal cognition (pp. 277. 289). Hillsdale, NJ: Erlbaum.

Blough, D. S. (1985). Discrimination of letters and random dot patterns by pigeons and humans. Journal of Experimental Psychology: Animal Behavior Processes, 11, 261-280.

Cerella, J. (1977). Absence of perspective processing in the pigeon. Pattern recognition, 9, 65-68.

DAvis, R. T. (1974). Monkeys as perceivers. In L. A. Rosenblum (Ed.), Primate behavior (Vol. 3). New York: Academic Press.

Dominguez, K. E. (1954). A study of visual illusions in the monkey. Journal of Genetic Psychology, 85, 105-127.

Donovan, W. J. (1978). Structure and function of the pigeon visual system. Physiological Psychology, 6, 403-437.

Fineman, M. B., \& Carlson, J. (1973). A comparison of the Ponzo 
illusion with a textural analogue. Perception \& Psychophysics, 14, 31-33.

Georgeson, M. A., Blakemore, C. (1973). Apparent depth and the Müller-Lyer illusion. Perception, 2, 225-234.

Glauber, M. (1986). Pigeon perception of a geometric illusion. Unpublished Master's Thesis, Hunter College, City University of New York.

GREGORY, R. L. (1963). Distortion of visual space as inappropriate constancy scaling. Nature, 199, 678-680.

HaRris, A. V. (1968). Perception of the horizontal-vertical illusion by stumptail monkeys. Radford Review, 22, 61-72.

Humphrey, N. K., \& Morgan, M. J. (1965). Constancy and the geometric illusions. Nature, 206, 744-745.

Malott, R. W., Malott, M. K. (1970). Perception and stimulus generalization. In W. C. Stebbins (Ed.), Animal psychophysics: The design and conduct of sensory experiments (pp. 363-400). New York: Plenum.
Malott, R. W., Malott, M. K., Pokrzywinski, J. (1967). The effects of outward-pointing arrowheads on the Müller-Lyer illusion in pigeons. Psychonomic Science, 9, 55-56.

Matsuzawa, T. (1985). Colour naming and classification in a chimpanzee (Pan troglodytes). Joumal of Human Evolution, 14, 283-291.

Newman, C. V., Newman, B. M. (1974). The Ponzo illusion in pictures with and without suggested depth. American Journal of Psychology, 87, 511-516.

Pressey, A. W. (1967). A theory of the Mueller-Lyer illusion. Perceptual \& Motor Skills, 25, 569-572.

Pressey, A. W. (1971). An extension of assimilation theory to illusions of size, area, and direction. Perception \& Psychophysics, 9, 172-176.

(Manuscript received January 14, 1991; revision accepted for publication May 15, 1991.) 